\title{
Treatable inherited metabolic epilepsies
}

\author{
Khalid Hundallah, MD, Brahim Tabarki, MD.
}

\begin{abstract}

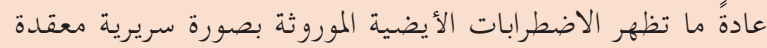

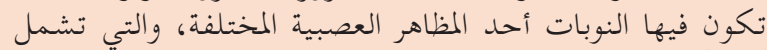

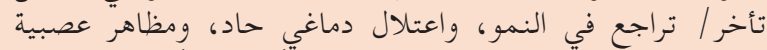

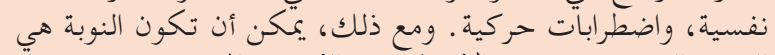

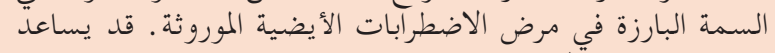

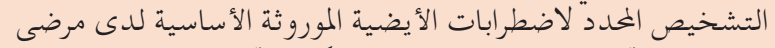

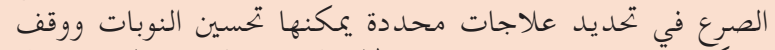

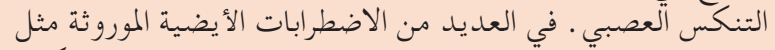

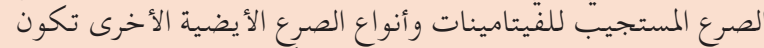

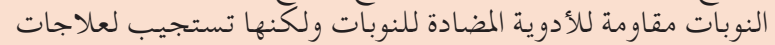

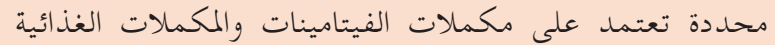

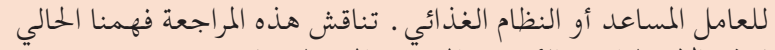

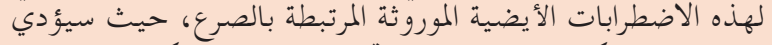

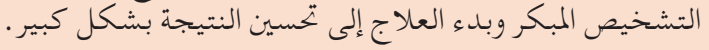

Inherited metabolic diseases usually present a complex clinical picture in which seizures are one of various neurological manifestations, which include developmental delays/regression, acute encephalopathy, neuropsychiatric manifestations, and movement disorders. However, a seizure can be the prominent feature in inherited metabolic disease. The specific diagnosis of an underlying inherited metabolic disorder in epileptic patients may help design specific treatments that can improve the seizures and stop neurodegeneration. In several inherited metabolic diseases such as vitamin-responsive epilepsies and other metabolic epilepsies, seizures are refractory to antiseizure medications but respond to specific treatments based on vitamin and cofactor supplementation or diet. This review discusses our current understanding of these inherited metabolic disorders associated with epilepsy, where early diagnosis and treatment initiation will significantly improve the outcome.

Neurosciences 2021; Vol. 26 (3): 229-235 doi: 10.17712/nsj.2021.3. 20210077

From the Division of Neurology, Department of Pediatrics, Prince Sultan Military Medical City, Riyadh, Kingdom of Saudi Arabia.

Address correspondence and reprint request to: Dr. Khalid Hundallah, Division of Neurology, Department of Pediatrics, Prince Sultan Military Medical City, Riyadh, Kingdom of Saudi Arabia. E-mail: dr.k.hundallah@gmail.com

ORCID ID: https://orcid.org/0000-0003-2570-8462
Therited metabolic diseases are common in highly consanguineous populations. ${ }^{1}$ They present with a wide range of neurological symptoms, including developmental delays/regression, seizures, acute encephalopathy, hypotonia, neuropsychiatric features, and movement disorders. ${ }^{2-4}$ Epilepsy is common in inherited metabolic diseases. It can present across one's lifespan and constitutes an immense challenge because it is usually refractory to the commonly used antiepileptic medications and impairs the quality of life of such patients. ${ }^{5-7}$ Some of these inherited metabolic diseases, such as vitamin-responsive epilepsies and other metabolic epilepsies, are amenable to specific treatments based on vitamin and cofactor supplementation or diet. Their clinical phenotypes, electroencephalographic (EEG) features, and magnetic resonance imaging (MRI) findings are mostly non-specific, but certain important clues can be obtained. Early diagnosis of an underlying treatable inherited metabolic disease as a cause of epilepsy is crucial since many will require specific management beyond common anti-seizure drugs, either to control seizures or to decrease the risk of brain injury. ${ }^{8}$ In this review, we discuss inherited metabolic disorders associated with epilepsy, where early diagnosis and treatment will significantly improve the outcome.

Pathophysiology of epilepsy in inherited metabolic diseases. The mechanisms of seizure generation in inherited metabolic disorders are diverse. ${ }^{9}$ They include accumulation of toxic metabolites (e.g., sulfocysteine), cofactor deficiency (e.g., vitamin B6), energy deficiency (e.g., glucose transporter 1(GLUT1)), substrate deficiency (e.g., serine biosynthesis deficiency), brain dysgenesis (e.g., pyruvate dehydrogenase deficiency), impaired neuronal function (e.g., storage disorder), disturbance of neurotransmitter systems (e.g., $\gamma$-aminobutyric acid [GABA] transaminase deficiency), or impaired metallation and transport (e.g., Menkes disease). ${ }^{9}$ Thus, a large proportion of inherited metabolic disorders can present with seizures, either during acute decompensation of the primary metabolic disorder or as a part of the complex phenotype of the inherited metabolic disorder. 
Common treatable inherited metabolic epilepsies. The common treatable inherited metabolic epilepsies are outlined in Table 1.

Pyridoxine-dependent epilepsy (PDE). PDE is inherited in an autosomal recessive pattern, and characterized by early-onset seizures refractory to antiseizure medications but responsive to pyridoxine (vitamin B6) supplementation. A variant causes it in the ALDH7A1 gene, which encodes alpha-aminoadipic semialdehyde dehydrogenase, also known as antiquitin, in the cerebral lysine degradation pathway. This leads to the accumulation of toxic metabolites in the central nervous system (alpha-aminoadipic semialdehyde, delta-1-piperideine-6-carboxylate, and pipecolic acid). ${ }^{10,11}$ Typically, seizures start in the neonatal period or the first few months of life. Multiple types of seizures can be observed, including tonic, partial, myoclonic, and prolonged episodes of status epilepticus. Infantile spasms and atonic seizures may also occur. EEG does not show any notable changes specific to the PDE. It may show diffuse slowing, multifocal discharges, or even burst suppression patterns. Several imaging abnormalities have been reported, such as thinning of the corpus callosum or intracerebral hemorrhage.

Biochemical testing reveals elevated alphaaminoadipic semialdehyde acid and pipecolic acid in body fluids, but both are non-specific. Molecular genetic testing of the ALDH7A1 gene is essential to confirm the diagnosis. More recently, mutations in PLPBP, a gene encoding a PLP-binding protein, have been reported as a novel finding in a minority of PDE.

Atypical features include late-onset (after the first year of life), prolonged seizure freedom despite being off pyridoxine, and seizures that initially respond to antiepileptic drugs but later become intractable.

Affected individuals require life-long supplementation of pyridoxine $(20-30 \mathrm{mg} / \mathrm{kg} /$ day) divided into 2 doses with a maximum of $500 \mathrm{mg} /$ day as higher doses may cause peripheral neuropathy. Dosage should be doubled during febrile illnesses. Treatment with pyridoxine must be combined with L-arginine or

Table 1 - Common inherited treatable metabolic epilepsies.

\begin{tabular}{|c|c|c|}
\hline IEM & Genetic causes & Treatments \\
\hline Pyridoxine dependent epilepsy & $\begin{array}{l}\text { ALDH7A1 } \\
\text { PROSC }\end{array}$ & $\begin{array}{c}\text { Pyridoxine }+/- \text { folinic acid } \\
\text { Arginine, Lysine restricted diet }\end{array}$ \\
\hline $\begin{array}{l}\text { Pyridox(am)ine } 50 \text {-Phosphate oxidase (PNPO) } \\
\text { Deficiency }\end{array}$ & PNPO & PLP +/- Pyridoxine \\
\hline Biotinidase deficiency & BTD & Biotin \\
\hline Glucose transporter 1 deficiency syndrome & SLC2A1 & Ketogenic diet \\
\hline Biotin-thiamine-responsive basal ganglia disease & SLC19A3 & Biotin, Thiamine \\
\hline Serine biosynthesis defects & PHGDH, PSAT1, PSPH & Serine + Glycine \\
\hline Molybdenum cofactor deficiency type - A & MOCS1 & cyclic pyranopterin monophosphate \\
\hline Cobalamin C deficiency & MMACHC & cobalamin, betaine \\
\hline Cerebral folate deficiency & FOLR1 & Folinic acid \\
\hline Creatine deficiency syndromes & $\begin{array}{l}\text { GAMT } \\
\text { GATM } \\
\text { SLC6A8 }\end{array}$ & $\begin{array}{l}\text { Creatine, arginine-restricted diet, ornithine } \\
\text { creatine monohydrate } \\
\text { creatine monohydrate }\end{array}$ \\
\hline Pyruvate dehydrogenase deficiency & $\begin{array}{l}\text { PDHA1 } \\
\text { PDHB, DLAT, PDHX, } \\
\text { PDP1 }\end{array}$ & Ketogenic diet \\
\hline $\begin{array}{l}\text { DEND (developmental delay, epilepsy, and neonatal } \\
\text { diabetes) syndrome }\end{array}$ & KCNJ11 & sulfonylurea \\
\hline Hyperinsulinism-Hyperammonemia syndrome & GLUD1 & Diazoxide + protein restriction \\
\hline $\begin{array}{l}\text { Tetrahydrobiopterin deficiency }(\mathrm{BH} 4) \\
\text { PTPS deficiency } \\
\text { DHPR deficiency } \\
\text { GTPCH deficiency } \\
\text { PCD } \\
\text { SPR deficiency }\end{array}$ & $\begin{array}{c}\text { PTS } \\
\text { QDPR } \\
\text { GCH1 } \\
\text { PCBD1 } \\
\text { SPR }\end{array}$ & $\begin{array}{l}\text { BH4, L-dopa, 5-HTP } \\
\text { Low phenylalanine diet, Folinic acid, L-dopa, } \\
\text { 5-HTP }\end{array}$ \\
\hline & & L-Dopa, 5-HTP \\
\hline
\end{tabular}


Table 2 - Main clinical, EEG, and MRI findings suggesting inherited metabolic epilepsies.

\begin{tabular}{|c|c|}
\hline \multicolumn{2}{|r|}{ Features } \\
\hline Onset & Early: neonatal, infancy or early childhood \\
\hline Seizure types & $\begin{array}{c}\text { Myoclonic, tonic, infantile spasms } \\
\text { Seizures worsening with fasting (GLUT1) or with high protein } \\
\text { meals (urea cycle defects) } \\
\text { Progressive myoclonic epilepsy phenotype } \\
\text { Seizures refractory to anti-epileptic drugs } \\
\text { Seizures worsening with anti-epileptic drugs }\end{array}$ \\
\hline Neurological features & $\begin{array}{c}\text { Developmental delay /regression } \\
\text { Movement disorders } \\
\text { Fluctuating course of illness }\end{array}$ \\
\hline Systemic symptoms & $\begin{array}{c}\text { Dysmorphic features } \\
\text { Organomegaly } \\
\begin{array}{c}\text { Ophthalmological abnormalities (cataracts, retinitis pigmentosa, } \\
\text { cherry red spot, optic nerve atrophy) }\end{array}\end{array}$ \\
\hline Family history & $\begin{array}{c}\text { Parental consanguinity } \\
\text { Metabolic or unexplained neurological disorder } \\
\text { Death of unknown etiology }\end{array}$ \\
\hline EEG & $\begin{array}{c}\text { Burst-suppression, multifocal spike discharges, hypsarrhythmia } \\
\text { Comb-like rhythm (maple syrup urine disease) }\end{array}$ \\
\hline MRI/MRS & $\begin{array}{l}\text { Normal / non specific abnormalities } \\
\text { Corpus callosum dysgenesis (pyruvate dehydrogenase deficiency) } \\
\text { Abnormal MRS in cerebral creatine deficiency or GABA transaminase deficiency }\end{array}$ \\
\hline
\end{tabular}

Table 3 - Suggested biochemical and genetic testing in early refractory seizures.

\begin{tabular}{lc}
\hline Source & Diagnostic test \\
\hline Blood & $\begin{array}{c}\text { Blood glucose, electrolytes, creatine, ammonia, lactic acid, uric acid, copper, ceruloplasmin, } \\
\text { homocysteine, biotinidase assay, VLCFA, transferrin isoelectric focusing, serum amino acids } \\
\text { alpha-aminoadipic semialdehyde acid, pipecolic acid, thiosulfate, xanthine, hypoxanthine, organic acids, } \\
\text { guanidinoacetate, creatine, creatinine. } \\
\text { Glucose, lactate, pyruvate, neurotransmitters }\end{array}$ \\
$\begin{array}{c}\text { Genetic testing } \\
\text { Array CGH, next generation sequencing (epilepsy panel, Whole exome sequencing) }\end{array}$ \\
\hline
\end{tabular}

lysine restriction diet to improve neurological outcomes. Arginine supplementation and a lysine-restricted diet could decrease lysine influx into the central nervous system and subsequently reduce the levels of these toxic intermediates..$^{10,11}$

Folinic acid-responsive epilepsy. Folinic acidresponsive seizures are allelic to PDE and have similar biochemical markers. Starting PDE patients on folinic acid at doses of $3-5 \mathrm{mg} / \mathrm{kg} /$ day could have clinical value in improving patients' conditions. ${ }^{12}$

Pyridox(am)ine 50 -phosphate oxidase (PNPO) deficiency. It is a rare autosomal recessive disorder caused by mutations in the PNPO gene, which leads to PNPO deficiency. This enzyme is essential for converting pyridoxine-5-phosphate and pyridoxamine5-phosphate into pyridoxal-5'-phosphate (PLP), which is the active form of pyridoxine. ${ }^{13}$ Typically, it is present in the neonatal period with refractory epilepsy similar to PDE, but it shows no or partial response to pyridoxine. Neonates have a wide range of non-neurological manifestations, such as prematurity, failure to thrive, hypoglycemia, lactic acidosis, and normocytic anemia. PNPO deficiency requires lifelong treatment with PLP $20-70 \mathrm{mg} / \mathrm{kg} /$ day divided into four doses. ${ }^{13}$

Biotinidase deficiency. It is an autosomal recessive disorder caused by mutations in the BTD gene. Biotinidase is essential for recycling biotin, necessary for biotin-dependent carboxylases to break down fats, proteins, and carbohydrates.

Biotinidase deficiency could be profound when biotinidase activity is reduced to less than $10 \%$ of the normal levels, or partial deficiency when biotinidase activity decreases to $10-30 \%$ of the normal. Without sufficient biotinidase, biotin cannot be recycled, which 
leads to the accumulation of toxic compounds in the body. ${ }^{14}$

It can present with various neurological symptoms, including hypotonia, seizures, alopecia, skin rash, visual impairment, and hearing loss. Multiple types of seizures were observed, including tonic, myoclonic, and spasms. EEG is nonspecific. The MRI shows diffuse or patchy signal abnormalities within the cerebral and cerebellar white matter, basal ganglia edema, and global atrophy.

Biotinidase deficiency responds to oral biotin 10-20 $\mathrm{mg}$ daily, resolving most of the symptoms, although hearing loss and visual impairment could persist. Screening for biotinidase deficiency is included in the national screening program in Saudi Arabia. ${ }^{14,15}$

Glut-1 deficiency. Glut-1 deficiency is a treatable disorder caused by impaired glucose uptake at the blood-brain barrier. It is caused by the SLC2A1 gene variant, which is essential in synthesizing glucose transporter protein type 1 , which affects the glucose transport across the blood-brain barrier, resulting in low cerebrospinal fluid glucose concentration. ${ }^{16}$

The phenotypes are variable. Typically, patients present with an early onset refractory epilepsy, developmental delay, and progressive microcephaly. SLC2A1 causes multiple seizure phenotypes, including focal seizures, generalized seizures, and infantile spasms, usually associated with irregular eye movements. Other types of epilepsy can be observed, including myoclonicastatic epilepsy, benign myoclonic epilepsy of infancy, and absence epilepsy. Unusual presentations include late-onset epilepsy and movement disorders.

The biochemical marker of Glut-1 deficiency is low cerebrospinal fluid glucose level $(<40 \mathrm{mg} / \mathrm{dL})$, cerebrospinal fluid glucose to blood glucose ratio $<0.4$, and low cerebrospinal fluid lactate level.

Prognosis is excellent should early diagnosis is established, and a ketogenic diet is started timely. Certain medications could compete with glucose at GLUT1 and worsen symptoms such as phenobarbital and valproic acid, and it is better to be avoided. ${ }^{15,16}$

Biotin-thiamine-responsive basal ganglia disease. It is an autosomal recessive disease caused by a variant of the SLC19A3 gene, which encodes hTHTR2, a second thiamine transporter. Typically, patients present with subacute encephalopathy, ataxia, ophthalmoplegia, extrapyramidal symptoms, and seizures. The phenotype was broadened to include early-onset Leigh-like syndrome, Wernicke-like encephalopathy, and infantile spasms. The brain MRI typically shows bilateral symmetric swelling in the caudate nucleus, putamen, and thalamus during the acute episode. Atrophy, necrosis and gliosis are observed at follow-up. Diagnosis is confirmed by sequencing the SLC19A3 gene. ${ }^{17}$

Once the diagnosis is suspected, initiate biotin (5 mg/kg/day) and thiamine (up to $1200 \mathrm{mg} /$ day) supplementation as early as possible. Symptoms typically resolve within 2-7 days. ${ }^{17}$

Serine biosynthesis defects. Serine biosynthesis defects result from deficiencies in the 3-phosphoglycerate dehydrogenase, phosphoserine aminotransferase, and phosphoserine phosphatase. Phenotypic presentations range from severe, lethal multiple congenital anomalies (Neu-Laxova syndrome) to milder phenotypes with an intellectual disability or refractory absence seizures in older children. ${ }^{15,18}$ An intermediate form exists wherein patients present with congenital microcephaly and refractory epilepsy in infancy. Early use of serine (400-600 mg/kg/day) and glycine (200-300 mg/ $\mathrm{kg} /$ day) improves seizure control and psychomotor outcomes. ${ }^{18}$

Molybdenum cofactor deficiency type - A (MOCOD). It is an autosomal recessive inherited disorder caused by mutations in the MOCS1 gene. Affected babies are normal at birth and develop refractory seizures and progressive encephalopathy in the first few weeks. MRI changes resemble those seen in hypoxic-ischemic injuries with cystic encephalomalacia. ${ }^{15,19}$

The MOCOD phenotypically and radiologically resembles sulfite oxidase deficiency. Biochemically, both show elevated urinary thiosulfate levels, but MOCOD also presents elevated urinary xanthine, hypoxanthine, and low serum uric acid levels. Early treatment with cyclic pyranopterin monophosphate improves patient outcomes. ${ }^{19}$

Cobalamin $C$ deficiency. It is an inherited defect in the MMACHC gene, which results in the dietary vitamin B12 not being converted to the active forms, methylcobalamin, and adenosylcobalamin. This results in the accumulation of methylmalonic acid and homocysteine and decreases methionine synthesis. Symptoms could start at any age. In the early onset, neurological symptoms begin in the 1st year of life with seizures, encephalopathy, hypotonia, and microcephaly. Hematologic, ocular, renal, hepatic, and cardiac symptoms are common. Initiating early treatment with vitamin $\mathrm{B} 12$ and betaine will improve outcomes. ${ }^{15,20}$

Cerebral folate deficiency. Cerebral folate deficiency is characterized by low levels of 5-methyltetrahydrofolate in the CSF despite normal folate levels in the plasma and red blood cells. This occurs secondary to FOLR1 gene mutations, autoantibodies against the folate receptor-alpha protein, or other conditions such as Rett syndrome and mitochondrial disorders. ${ }^{15,21}$ 
Symptoms typically start in infancy with hypotonia, progressive microcephaly, choreoathetoid movements, and seizures. High-dose folinic acid treatment (1-5 mg/ $\mathrm{kg} /$ day) ameliorates the neurological symptoms. ${ }^{21}$

Creatine deficiency syndromes. It is an inborn error of creatine metabolism which interrupts the synthesis or transportation of creatine, which is essential for sustaining the high energy levels required for muscle and brain development. There are three forms of 2 autosomal recessive biosynthesis defects: guanidinoacetate methyltransferase deficiency (GAMT) and arginine: glycine amidinotransferase deficiency (AGAT) and an X-linked creatine transporter deficiency (CTD). ${ }^{15,22}$ Symptoms include developmental delay, intellectual disability, hypotonia, autistic symptoms, movement disorders, and seizures.

The MR spectroscopy shows depletion of the cerebral creatine in all individuals with GAMT and AGAT deficiency and men with CTD. Biochemical screening is performed by measuring guanidinoacetate, creatine, and creatinine in urine and plasma. Diagnosis is confirmed by molecular testing of the 3 genes involved (GAMT, GATM, and SLC6A8). Treatment of patients with AGAT or GAMT deficiency is based on supplementation of creatine monohydrate (350-2000 $\mathrm{mg} / \mathrm{kg} /$ day). Children with GAMT need to also be placed on an arginine-restricted diet to reduce high levels of neurotoxic guanidinoacetate, and also started on ornithine. However, children with CTD are less responsive to therapy. ${ }^{22}$

The DEND syndrome (developmental delay, epilepsy, and neonatal diabetes). DEND syndrome is a rare inherited disorder caused by mutations in the ATPsensitive potassium channel (KATP channel) subunits, which leads to the inhibition of KATP pathwaydependent insulin release in pancreatic cells and inhibition of neurotransmitter release. Symptoms start early in the neonatal period with neonatal diabetes and dysmorphic features. Neurological features, including hypotonia, developmental delay, and refractory seizures, are common. Different types of seizures can be observed, such as tonic, myoclonic, and infantile spasms. Treatment with oral sulfonylurea blocks the KATP channel restores insulin secretion and improves glycemic control and seizures. ${ }^{23}$

"Hyperinsulinism-Hyperammonemia" syndrome. Hyperinsulinism-hyperammonemia syndrome is a rare inherited condition characterized by excessive insulin secretion, causing repeated episodes of hypoglycemia and abnormal elevation in serum ammonia. It is caused by mutations in the GLUD1 gene, which results in increased activity of the mitochondrial enzyme glutamate dehydrogenase. This leads to increased conversion of glutamate to alpha-ketoglutarate, with subsequent adenosine triphosphate (ATP) generation, leading to KATP-related insulin release and affecting the detoxification of ammonia in the liver. Common neurological symptoms include seizures, behavioral disorders, and developmental delay. Seizures could be secondary to hypoglycemia, but also primarily generalized epilepsy is possible. ${ }^{15,24}$

Treatment involves starting diazoxide $(5-15 \mathrm{mg} /$ $\mathrm{kg} /$ day), dietary protein restriction, and intermittent glucagon administration for hypoglycemic crises. ${ }^{24}$

Tetrahydrobiopterin (BH4) deficiency. It is a group of inherited metabolic disorders affecting the biosynthesis and regeneration of $\mathrm{BH} 4$, which causes increased serum phenylalanine levels. $\mathrm{BH} 4$ is essential for the production of dopamine, epinephrine, norepinephrine, and serotonin. There are 5 main forms of $\mathrm{BH} 4$ deficiency, 3 biosynthesis defects; guanosine triphosphate cyclohydrolase I deficiency, 6-pyruvoyl tetrahydropterin synthase (PTPS) deficiency, and sepiapterin reductase (SR) deficiency and 2 regeneration defects; pterin-4-alpha-carbinolamine dehydratase deficiency and dihydropteridine reductase (DHPR) deficiency. PTPS defects are the most common cause. Sepiapterin reductase deficiency is the only one that does not elevate phenylalanine. ${ }^{15,25}$

Symptoms start in the 1 st few months of life with dystonia, abnormal muscle tone, and seizures. Symptoms are progressive, leading to progressive microcephaly, global developmental delay, and intellectual disability. The SR deficiency presents with dystonia, oculogyric crises, and developmental delay mimicking cerebral palsy. The DHPR-related deficiency leads to $\mathrm{BH} 2$ accumulation, causing a severe form of the disease and resulting in cerebral folate deficiency and calcifications in the basal ganglia. The neurodevelopmental outcome is correlated with the early initiation of treatment with a low-phenylalanine diet, BH4, L-dopa, and 5-hydroxytryptophan. Starting with carbidopa, monoamine oxidase $\mathrm{B}$, and catechol-Omethyltransferase inhibitors reduce L-dopa's therapeutic requirements and its peripheral adverse effects.

DHPR deficiency could lead to cerebral folate deficiency; therefore, folinic acid should be administered, and it is better to avoid $\mathrm{BH} 4$ since it will augment cerebral folate deficiency. 15,25

Pyruvate dehydrogenase deficiency (PPD). The PPD is a mitochondrial disorder that affects ATP production and causes the accumulation of lactic acid. It causes progressive neurological symptoms, typically in infancy. Symptoms include abnormal muscle tone, 
developmental delay, abnormal eye movements, and seizures.

Cofactor supplementation with thiamine, carnitine, and lipoic acid stimulates the pyruvate dehydrogenase complex to produce energy. A ketogenic diet (KD) should be started early since it can bypass metabolic defects and control seizures. The KD is effective and safe for controlling seizures during PDD. It should be introduced as early as possible upon diagnosis, as early initiation may prevent further metabolic damage to the brain. ${ }^{15,26}$

Succinic semialdehyde dehydrogenase deficiency. It is a rare monogenic disorder caused by a mutation in the ALDH5A1 gene. This will impair the activity of the mitochondrial enzyme succinic semialdehyde dehydrogenase, which will, in turn, affect the GABA metabolism. Clinical manifestations vary significantly and include intellectual disability/developmental delay, autistic symptoms, hypotonia, and seizures. Different types of seizures could be observed, mainly tonicclonic, atypical absences, and myoclonic seizures. ${ }^{15,27}$ Detection of GABA in urine is a useful screening tool for this disease. A broad spectrum of antiepileptic drugs is required to control seizures, mainly sodium channel blockers, such as lamotrigine and carbamazepine. Other therapies, such as vigabatrin and mTOR inhibitors, are exciting treatment options. Several new therapies are still under investigation, including enzyme replacement therapy, chaperones, read-through drugs, and gene therapy. ${ }^{27}$

Neuronal ceroid lipofuscinosis type 2. Neuronal ceroid lipofuscinosis type 2 is a rare neurodegenerative lysosomal storage disorder caused by a deficient tripeptidyl peptidase 1 (TPP1) enzyme. It typically manifests between 2 and 4 years of age with seizures, language delay/arrest, and rapid psychomotor regression. ${ }^{15,28}$ Seizures are usually polymorphic, including multiple types of seizures, and resistant to anti-seizures drugs. EEG with low-frequency $(1-3 \mathrm{~Hz})$ intermittent photic stimulation shows a characteristic photoparoxysmal response. Diagnosis of neuronal ceroid lipofuscinosis type 2 should be based on genetic testing for mutations in TPP1 and enzyme activity testing of TPP1 protein. Intracerebroventricular administration of cerliponase alfa (rhTPP1 enzyme) stabilizes neuronal ceroid lipofuscinosis type 2 progression, adding this disease to the list of potentially treatable disorders requiring prompt diagnosis. ${ }^{28}$

Approach to treatable inherited metabolic epilepsies. The presence of any of sign and symptom highlighted in Table 2 should raise suspicion for an underlying metabolic etiology of epilepsy. The expression of the majority of inherited metabolic epilepsies is in the neonatal period or early infancy, although late presentation until adulthood has been reported. Taking a detailed history, paying attention to family history, and performing a thorough clinical examination in all patients presenting with early-onset seizures allows establishing an early diagnosis. Neonates and infants presenting with refractory seizures should be started on pyridoxine, folinic acid, and biotin to avoid therapeutic delay until the biochemical/or genetic workup results for inherited metabolic diseases are available. ${ }^{29}$

The work-up of a patient with a suspected inherited metabolic disease as a cause of epilepsy is based on a combination of EEG, neuroimaging, biochemical and molecular genetic testing (Tables 2 and 3 ). The presence of specific EEG or brain neuroimaging findings can provide important clues toward the correct diagnosis. EEG helps to clarify the seizure type and type of epileptic encephalopathy. Some inherited metabolic diseases can have characteristic EEG changes, such as the comb-like rhythm seen in patients with maple syrup urine disease. Imaging findings sometimes suggest a particular inherited metabolic disease, as is the case with thiamine-biotin-responsive basal ganglia disease, cerebral creatine deficiency, GABA transaminase deficiency, or molybdenum cofactor deficiency, but more often show non-specific changes (e.g., dysgenesis of the corpus callosum), and normal brain structure and development, which do not exclude an inherited metabolic disease. The laboratory and genetic testing results are outlined in Table 3. Recently, a new LC-MS/ MS method, replacing individual metabolite testing assays with a novel "panel-method," greatly improves the biochemical diagnostic workup of children with early-onset treatable inherited metabolic epilepsies. ${ }^{29}$

Conclusion. A growing number of treatable inherited metabolic disorders associated with epilepsy have been described. Rapid diagnosis and early initiation of appropriate treatment are essential to avoid or minimize irreversible brain damage. Various epilepsy phenotypes can be observed, often refractory to antiepileptic drugs. A high index of suspicion is required to make a diagnosis because the clinical presentation is often non-specific. Early diagnosis and treatment are associated with better outcomes.

Acknowledgements. The authors would like to thank Editage company (www.editage.com) for English language editing.

\section{References}

1. Afzal RM, Lund AM, Skovby F. The impact of consanguinity on the frequency of inborn errors of metabolism. Mol Genet Metab Rep 2018; 15: 6-10. 
2. BoAli AY, Alfadhel M, Tabarki B. Neurometabolic disorders and congenital malformations of the central nervous system. Neurosciences (Riyadh) 2018; 23: 97-103.

3. Hakami WS, Hundallah KJ, Tabarki BM. Metabolic and genetic disorders mimicking cerebral palsy. Neurosciences (Riyadh) 2019; 24: 155-163.

4. Assi L, Saklawi Y, Karam PE, Obeid M. Treatable Genetic Metabolic Epilepsies. Curr Treat Options Neurol 2017; 19: 30.

5. Bashiri FA, Al-Sehemi MA, Hamad MH, Alshammari NR, Aljumah MA, Kentab A, et al. Neurodevelopmental and epilepsy outcomes of patients with infantile spasms treated in a tertiary care center. Neurosciences (Riyadh) 2021; 26: 21-25.

6. Alyoubi RA, Aljaafari DT, Basheikh MA, Al-Yahyawi NY, Bakry MA, BenHli NM, et al. The etiology and risk factors of convulsive status epilepticus in pediatric patients of tertiary center in Saudi Arabia. Neurosciences (Riyadh) 2021; 26: 26-30.

7. Al-Qudah AA, Albsoul-Younes A, Masri AT, AbuRahmah SK, Alabadi IA, Nafi OA, et al. Type and etiology of pediatric epilepsy in Jordan. Neurosciences (Riyadh) 2017; 22: 267-273.

8. Muthaffar OY. Treating epilepsy with options other than antiepileptic medications. Neurosciences (Riyadh) 2020; 25: 253-261.

9. Dulac O, Plecko B, Gataullina S, Wolf NI. Occasional seizures, epilepsy, and inborn errors of metabolism. Lancet Neurol 2014; 13: 727-739.

10. Al-Saman AS, Rizk TM. A case of extreme prematurity and delayed diagnosis of pyridoxine-dependent epilepsy. Neurosciences (Riyadh) 2012; 17: 371-373.

11. Coughlin CR 2nd, Tseng LA, Abdenur JE, Ashmore C, Boemer F, Bok LA, et al. Consensus guidelines for the diagnosis and management of pyridoxine-dependent epilepsy due to alphaaminoadipic semialdehyde dehydrogenase deficiency. J Inherit Metab Dis 2021; 44: 178-192.

12. Gallagher RC, Van Hove JL, Scharer G, Hyland K, Plecko B, Waters PJ, et al. Folinic acid-responsive seizures are identical to pyridoxine-dependent epilepsy. Ann Neurol 2009; 65: $550-556$

13. Alghamdi M, Bashiri FA, Abdelhakim M, Adly N, Jamjoom DZ, Sumaily KM, et al. Phenotypic and molecular spectrum of pyridoxamine-5'-phosphate oxidase deficiency: A scoping review of 87 cases of pyridoxamine-5'-phosphate oxidase deficiency. Clin Genet 2021; 99: 99-110.

14 Sayegh LN, Daher RT, Bassyouni A, Karam PE. Diagnosis and management of symptomatic profound biotinidase deficiency in a tertiary care center in Lebanon. Clin Biochem 2020; 86: 52-55.

15. Van Karnebeek CDM, Sayson B, Lee JJY, Tseng LA, Blau N, Horvath GA, et al. Metabolic Evaluation of Epilepsy: A Diagnostic Algorithm With Focus on Treatable Conditions. Front Neurol 2018; 9: 1016.

16. Klepper J, Akman C, Armeno M, Auvin S, Cervenka M, Cross HJ, et al. Glut1 Deficiency Syndrome (Glut1DS): State of the art in 2020 and recommendations of the international Glut1DS study group. Epilepsia Open 2020; 5: 354-365.
17. Tabarki B, Al-Shafi S, Al-Shahwan S, Azmat Z, Al-Hashem A, Al-Adwani N, et al. Biotin-responsive basal ganglia disease revisited: clinical, radiologic, and genetic findings. Neurology 2013; 80: 261-267.

18. El-Hattab AW. Serine biosynthesis and transport defects. Mol Genet Metab 2016; 118: 153-159.

19. Schwahn BC, Van Spronsen FJ, Belaidi AA, Bowhay S, Christodoulou J, Derks TG, et al. Efficacy and safety of cyclic pyranopterin monophosphate substitution in severe molybdenum cofactor deficiency type A: a prospective cohort study. Lancet 2015; 386: 1955-1963.

20. He R, Mo R, Shen M, Kang L, Song J, Liu Y, et al. Variable phenotypes and outcomes associated with the MMACHC c.609G $>\mathrm{A}$ homologous mutation: long term follow-up in a large cohort of cases. Orphanet J Rare Dis 2020;15:200.

21. Al-Baradie RS, Chudary MW. Diagnosis and management of cerebral folate deficiency. Neurosciences (Riyadh) 2014; 19: 312-316.

22. Fons C, Campistol J. Creatine Defects and Central Nervous System. Semin Pediatr Neurol 2016; 23: 285-289.

23. Letourneau LR, Greeley SAW. Precision Medicine: Long-Term Treatment with Sulfonylureas in Patients with Neonatal Diabetes Due to KCNJ11 Mutations. Curr Diab Rep 2019; 19: 52.

24. Ninković D, Sarnavka V, Bašnec A, Ćuk M, Ramadža DP, Fumić K, et al. Hyperinsulinism-hyperammonemia syndrome: a de novo mutation of the GLUD1 gene in twins and a review of the literature. J Pediatr Endocrinol Metab 2016; 29: 1083-1088.

25. Opladen T, López-Laso E, Cortès-Saladelafont E, Pearson TS, Sivri HS, Yildiz Y, et al. Consensus guideline for the diagnosis and treatment of tetrahydrobiopterin (BH4) deficiencies. Orphanet J Rare Dis 2020; 15: 126.

26. Matricardi S, Canafoglia L, Ardissone A, Moroni I, Ragona F, Ghezzi D, et al. Epileptic phenotypes in children with early-onset mitochondrial diseases. Acta Neurol Scand 2019; 140: 184-193.

27. Horino A, Kawawaki H, Fukuoka M, Tsuji H, Hattori Y, Inoue $\mathrm{T}$, et al. A case of succinic semialdehyde dehydrogenase deficiency with status epilepticus and rapid regression. Brain Dev 2016; 38: 866-870.

28. Mazurkiewicz-Bełdzińska M, Del Toro M, Haliloğlu G, Huidekoper HH, Kravljanac R, Mühlhausen C, et al. Managing CLN2 disease: A treatable neurodegenerative condition among other treatable early childhood epilepsies. Expert Rev Neurother 2021; 1-8.

29. Mathis D, Beese K, Rüegg C, Plecko B, Hersberger M. LC-MS/MS method for the differential diagnosis of treatable early onset inherited metabolic epilepsies. J Inherit Metab Dis 2020; 43: 1102-1111. 